\title{
Fast Spin-Flip Enables Efficient and Stable Organic Electroluminescence from Charge-Transfer States
}

Lin-Song Cui ${ }^{1, \#}$, Alexander J. Gillett ${ }^{1, \#}$, Shou-Feng Zhang ${ }^{2,3, \#}$, Hao Ye ${ }^{4}$, Yuan Liu ${ }^{5}$, Xian-Kai Chen $^{3, *}$, Ze-Sen Lin ${ }^{4}$, Emrys W. Evans ${ }^{1}$, William K. Myers ${ }^{6}$, Tanya K. Ronson ${ }^{7}$, Hajime Nakanotani $^{4}$, Sebastian Reineke ${ }^{5}$, Jean-Luc Bredas ${ }^{3}$, Chihaya Adachi ${ }^{4,}$, Richard H. Friend ${ }^{1, *}$

${ }^{1}$ Cavendish Laboratory, University of Cambridge, JJ Thomson Avenue, Cambridge, CB3 OHE, United Kingdom.

${ }^{2}$ Department of Electronic Engineering, Guangxi University of Science and Technology, Liuzhou 545006, China

${ }^{3}$ School of Chemistry and Biochemistry, Center for Organic Photonics and Electronics, Georgia Institute of Technology, Atlanta, GA, 30332-0400, USA

${ }^{4}$ Center for Organic Photonics and Electronics Research (OPERA), Kyushu University, 744 Motooka, Nishi, Fukuoka 819-0395, Japan.

${ }^{5}$ Dresden Integrated Center for Applied Physics and Photonic Materials (IAPP), Technische Universität Dresden, Dresden, 01069, Germany.

${ }^{6}$ Centre for Advanced Electron Spin Resonance (CAESR), University of Oxford, South Parks Road, Oxford, OX1 3QR, UK.

${ }^{7}$ Department of Chemistry, University of Cambridge, Lensfield Road, Cambridge, CB1EW, United Kingdom.

\# These authors contributed equally to this work.

* Authors to whom correspondence should be addressed:

E-mail: rhf10@cam.ac.uk (RHF); adachi@cstf.kyushu-u.ac.jp (AC); chenxiankai@email.arizona.edu (XKC) 
A spin-flip from a triplet to a singlet excited state, that is, reverse intersystem crossing (RISC), is an attractive route to harvest triplet excitons for light emission in organic lightemitting diodes (OLEDs). High external quantum efficiencies (EQE) have been obtained via thermally activated delayed fluorescence (TADF) that exploits this RISC mechanism. However, device stability and efficiency roll-off remain challenging issues that originate from the slow RISC rate $\left(k_{\mathrm{RISC}}\right)$, with the resulting long triplet exciton lifetime leading to severe exciton annihilation processes. Here, we demonstrate that the introduction of multiple donor units that form charge-resonance-type hybrid triplet states leads to a small singlet-triplet energy splitting, large spin-orbit couplings, and a dense manifold of triplet states energetically close to the singlet state. Because of these attributes, $k_{\mathrm{RISC}}$ in our novel TADF molecule is as fast as $1.5 \times 10^{7} \mathrm{~s}^{-1}$ : a value some two orders of magnitude higher than those in typical TADF emitters. OLEDs based on this molecule serving as an emitter or a sensitizer exhibit long-term stability (the time to reach $90 \%$ of the initial brightness of $1000 \mathrm{~cd} \mathrm{~m}^{-2}$ is estimated to be about 600 hours), very high efficiency $\left(\mathrm{EQE}_{\max }>\mathbf{2 9 . 3 \%}\right)$, and a remarkably low efficiency roll-off $(<2.3 \% \mathrm{EQE}$ roll-off at $1,000 \mathrm{~cd} \mathrm{~m}^{-2}$ ). Our work offers guidelines to design purely organic molecules with a fast $k_{\text {RISC, }}$ which will ultimately facilitate OLED applications exploiting the TADF mechanism. 


\section{Main}

The triplet excited states of organic $\pi$-conjugated molecules play an essential role in organic optoelectronic devices, such as organic light-emitting diodes (OLEDs), organic solar cells, and organic semiconductor lasers. ${ }^{1,2,3,4}$ Efficiently harvesting triplet excitons is critical in OLEDs, since spin-statistical charge recombination leads to the formation of triplet to singlet excitons in a 3:1 ratio. ${ }^{5,6,7,8,9,10,11,12}$ Recent developments have shown that purely organic emitters exploiting thermally activated delayed fluorescence (TADF) in OLEDs can achieve nearly 100\% internal quantum efficiencies (IQE). ${ }^{13,14,15,16,17}$ However, severe issues still plague TADFbased OLEDs: for example, operational stability and efficiency roll-off remain unsatisfactory, which largely limits their potential for commercialization. ${ }^{18,19,20,21}$

In TADF emitters, a fast reverse intersystem crossing (RISC) process from the triplet to the singlet state is crucial to the efficient utilization of triplet excitons. Early on, RISC was considered to be a process involving simply the lowest triplet $\left(\mathrm{T}_{1}\right)$ state and the lowest singlet $\left(\mathrm{S}_{1}\right)$ excited state: the degree $(\alpha)$ of mixing of these states can be expressed in the framework of first-order perturbation theory as $\alpha \propto S O C_{S_{1}-T_{1}} / \Delta E_{S_{1}-T_{1}}$, where $S O C_{S_{1}-T_{1}}$ and $\Delta E_{S_{1}-T_{1}}$ denote the spin-orbit coupling (SOC) between the $S_{1}$ and $T_{1}$ states and their energy gap, respectively. ${ }^{22}$ This relationship suggests small $\Delta E_{S_{1}-T_{1}}$ and large $S O C_{S_{1}-T_{1}}$ values are required in order to facilitate efficient RISC. Over the past decade, small $\Delta E_{S_{1}-T_{1}}($ e.g.,$<0.1 \mathrm{eV})$ values have been reported in a broad series of simple donor-acceptor (D-A)-type molecules: in such instances, the spatial separation between the highest occupied and lowest unoccupied molecular orbitals (HOMO and LUMO) leads to very small electron-exchange energy (equal to $\Delta E_{S_{1}-T_{1}} / 2$ when the main electronic configuration describing both the $\mathrm{S}_{1}$ and $\mathrm{T}_{1}$ states simply corresponds to a HOMO-to-LUMO transition). ${ }^{23,24,25,26,27,28,29,30,31,32,33}$ In such D-A-type molecules, however, the $S_{1}$ and $T_{1}$ states often have a dominant charge-transfer (CT) -exciton 
character, which results in a vanishingly small SOC $\left(e . g\right.$. , on the order of $\left.<0.1 \mathrm{~cm}^{-1}\right)$ between the two CT states. ${ }^{34}$ The consequence of this negligible SOC is that RISC from a CT-type $\mathrm{T}_{1}$ to a CT-type $S_{1}$ is inefficient even when $\Delta E_{S_{1}-T_{1}}$ is small, leading to rate constants ( $k_{\mathrm{RISC}}$ ) in most of the reported TADF emitters lower than $10^{6} \mathrm{~s}^{-1} \cdot 35,36,37,38$ The negative outcome of a slow RISC process is long triplet exciton lifetimes, resulting in bimolecular exciton annihilation processes that are responsible for the severe efficiency roll-offs and material degradation occurring in TADF-based OLEDs. ${ }^{39,40,41,42,43}$ Thus, designing novel TADF molecules with fast RISC rates is highly desirable to improve OLED stability and efficiency.

Our earlier theoretical investigations on Cz-TRZ (9-(4-(4,6-diphenyl-1,3,5-triazine-2yl)phenyl)-9H-carbazole) and its derivatives indicated that the $\mathrm{T}_{1}$-state wavefunctions in these molecules are confined to the carbazole-phenylene-triazine fragment. ${ }^{44}$ Importantly, it was predicted that the introduction of additional carbazole donors (which have a high-energy triplet state) would decrease the $S_{1}$-state energies but would have little impact on the $T_{1}$-state energies and wavefunctions: the consequence is not only a reduced $\Delta E_{S_{1}-T_{1}}$ value but also $\mathrm{S}_{1}$ and $\mathrm{T}_{1}$ states with different excitation characters. ${ }^{44}$ Additionally, we have shown that in D-A-type TADF molecules, the introduction of multiple donor moieties that result in the formation of charge-resonance-type hybrid triplet states leads to the appearance of a dense manifold of triplet states. This opens up additional RISC transition channels from higher-lying triplet (e.g., $T_{2}$ ) states to $S_{1}$ (via a second-order spin-vibronic mechanism), which eventually facilitates the spin-flip processes. ${ }^{45,46}$

Based on these earlier findings, ${ }^{44,45,46}$ we have designed and synthesized a novel TADF emitter consisting of five carbazole $(\mathrm{Cz})$ donors and one triazine (TRZ) acceptor, i.e., 5Cz-TRZ (9,9',9",9'",9'"'-(6-(4,6-diphenyl-1,3,5-triazine-2-yl)benzene-1,2,3,4,5-pentayl)pentakis(9Hcarbazole)), see Fig. 1a. For the sake of comparison, we have also investigated three other molecules: mCz-TRZ (9-(4-(4,6-diphenyl-1,3,5-triazin-2-yl)phenyl)-3,6-dimethyl-9H- 
carbazole), TmCz-TRZ (9,9',9"-(5-(4,6-diphenyl-1,3,5-triazine-2-yl)benzene-1,2,3-triyl) tris(3,6-dimethyl-9H-carbazole)), and DACT-II (9-[4-(4,6-diphenyl-1,3,5-triazine-2yl)phenyl]-N,N,N0,N0-tetraphenyl-9H-carbazole- 3,6-diamine)), see Fig. 1a. We demonstrate below that in addition to the appearance of a dense triplet-state manifold, a small $\Delta E_{S_{1}-T_{1}}$ and a large spin-orbit coupling are simultaneously achieved in 5Cz-TRZ. As a result, 5Cz-TRZ shows an ultra-fast $k_{\mathrm{RISC}}, \sim 1.5 \times 10^{7} \mathrm{~s}^{-1}$, a value some two orders magnitude faster than those found in the conventional TADF molecules. ${ }^{16,17,19,21,28}$ Such a fast RISC process reduces the triplet exciton concentration and the number of bimolecular annihilation events in the emission layer, which in-turn increases the lifetimes of the TADF-based OLEDs more than tenfold and overcomes the efficiency roll-off issue. Furthermore, full-colour hyperfluorescent OLEDs exploiting 5Cz-TRZ as a sensitizer also exhibit very high EQE values of over $24.9 \%$. Our achievement of a fast $k_{\text {RISC }}\left(>10^{7} \mathrm{~s}^{-1}\right)$ in purely organic molecules opens the door to highly efficient and stable TADF-based OLEDs.

\section{Results and discussion}

\section{Computational Results}

As shown by the Natural Transition Orbital (NTO) analysis in Fig. 1b, the $S_{1}$ state of 5Cz-TRZ shows a pronounced CT-excitation character; the hole and electron wavefunctions are localized at the ortho- and meta-carbazole donors and the central (para-carbazole)-phenylene-triazine, respectively, with a small spatial overlap $\left(\mathrm{O}_{\mathrm{h} / \mathrm{e}}\right)$ of $\sim 0.3$. In contrast, the $\mathrm{T}_{1}$ state shows a hybrid character as it consists of local and CT excitations with a large $\mathrm{O}_{\mathrm{h} / \mathrm{e}}$ of $\sim 0.7$. (see the "Computational Details" section for more details on the calculations). The marked difference in the excitation characters of the $S_{1}$ and $T_{1}$ states results in a significant $S O C_{S_{1}-T_{1}}$ value of $\sim$ $0.4 \mathrm{~cm}^{-1}$. Intriguingly, the calculations predict a $\Delta E_{S_{1}-T_{1}}$ value as small as $\sim 0.02 \mathrm{eV}$, which is usually obtained in case where $\mathrm{S}_{1}$ and $\mathrm{T}_{1}$ both have CT character. The central (para-carbazole)- 
phenylene-triazine present in 5Cz-TRZ has a low-energy triplet state at $\sim 2.92 \mathrm{eV}$ due to the large electronic coupling between the para-carbazole and phenylene-triazine fragments, see Fig. 1c. The additional ortho- and meta-carbazole donors in $5 \mathrm{Cz}-\mathrm{TRZ}$ possess higher-energy triplet states $(\sim 3.61 \mathrm{eV})$, which have little effect on the $\mathrm{T}_{1}$-state energy $\left(\sim 2.95 \mathrm{eV}\right.$ at the $\mathrm{T}_{1}$-state equilibrium geometry) of the whole $5 \mathrm{Cz}$-TRZ molecule (Fig. 1c), a result consistent with our earlier calculations. ${ }^{44}$ In contrast, the additional donors largely stabilize the $S_{1}$-state energy of 5Cz-TRZ due to electronic delocalization and polarization effects. The consequence is the realization of both a large $S O C_{S_{1}-T_{1}}$ and a small $\Delta E_{S_{1}-T_{1}}$ value in $5 \mathrm{Cz}$-TRZ. In addition, as demonstrated in our earlier investigations, ${ }^{46}$ the $\mathrm{Cz}$ donors connected to the phenylene-triazine acceptor leads to a dense manifold of triplet states (with a small energy gap between $\mathrm{T}_{1}$ and $\mathrm{T}_{2}$, $\sim 0.24 \mathrm{eV}$ ), due to the formation of charge-resonance-type hybrid triplet states, see Fig. $\mathbf{1 b}$. Another feature is that the $S O C_{S_{1}-T_{2}}$ (SOC between the $T_{2}$ and $S_{1}$ states, $\sim 1.3 \mathrm{~cm}^{-1}$ ) is significantly larger than $S O C_{S_{1}-T_{1}}$, a consequence of the differences in the spatial distributions of the $\mathrm{T}_{1}$ and $\mathrm{T}_{2}$ hole wavefunctions (Fig. 1b). In the framework of Marcus electron-transfer theory, the rate constants from the $\mathrm{T}_{1}$ and $\mathrm{T}_{2}$ to $\mathrm{S}_{1}\left(k_{T_{1} \rightarrow S_{1}}\right.$ and $\left.k_{T_{2} \rightarrow S_{1}}\right)$ are evaluated to be 4.1 $\times 10^{6}$ and $1.4 \times 10^{9} \mathrm{~s}^{-1}$, respectively. Importantly, the small energy gap between the $\mathrm{T}_{1}$ and $\mathrm{T}_{2}$ states opens a spin-flip channel from $T_{2}$ to $S_{1}$ in addition to the $T_{1}$-to- $S_{1}$ channel, which implies that the RISC rate in $5 \mathrm{Cz}-\mathrm{TRZ}$ must be larger than $4.1 \times 10^{6} \mathrm{~s}^{-1}$.

It is obvious from the above discussion that our current molecular-design strategy for 5Cz-TRZ is based on two ideas: (i) Whilst the $\mathrm{T}_{1}$ exciton is confined in the (para-carbazole)phenylene-triazine segment and has a low triplet energy, the introduction of the additional $\mathrm{Cz}$ donors in the ortho- and meta-positions significantly decreases the $\mathrm{S}_{1}$-state energy, which ultimately reduces the $\Delta E_{S_{1}-T_{1}}$ value. Additionally, since we have confined the $\mathrm{T}_{1}$ state, it now possesses a local-excitation (LE) character, whilst the $\mathrm{S}_{1}$ state has a CT character; as a result the spin-orbit coupling between the LE-dominated $\mathrm{T}_{1}$ state and the CT-dominated $\mathrm{S}_{1}$ state is 
strong. (ii) The introduction of the additional $\mathrm{Cz}$ donors in the ortho- and meta-positions leads to another positive characteristic, i.e., a dense manifold of triplet states due to the formation of charge-resonance-type hybrid triplet states, which opens up the $\mathrm{T}_{2}$-to- $\mathrm{S}_{1}$ RISC channel, in addition to the $\mathrm{T}_{1}$-to- $\mathrm{S}_{1}$ channel.

The data for the other three molecules (mCz-TRZ, TmCz-TRZ, and DACT-II) we examined for the sake of comparison are given in Supplementary Fig. S1 and Table S1. In these molecules, the absence of multiple donor groups that could form charge-resonance-type hybrid triplet states results in RISC rates that are 1 to 4 orders of magnitude slower than in 5Cz-TRZ, which significantly hinders their electroluminescent performance and device stability.

\section{Photophysical Properties}

The photophysical properties of 5Cz-TRZ were analysed using ultraviolet-visible (UV/Vis) and photoluminescence (PL) spectroscopies. As shown in Fig. 2a, the absorption spectrum of 5Cz-TRZ is formed from the combination of the $\mathrm{Cz}$ donor and TRZ acceptor absorptions, with an additional weak absorption band between $380-420 \mathrm{~nm}$ that is attributed to the intramolecular CT transition from the $\mathrm{Cz}$ donors to the TRZ acceptor. With increasing solvent polarity, the emission spectrum of $5 \mathrm{Cz}$-TRZ was observed to broaden and red-shift from 470 $\mathrm{nm}$ (cyclohexane) to $550 \mathrm{~nm}$ (dimethyl formamide), which is consistent with the strong CT character of its first singlet excited state. Fig. 2b displays the PL and phosphorescence (Phos) spectrum of $5 \mathrm{Cz}-\mathrm{TRZ}$ in a frozen toluene matrix at $77 \mathrm{~K}$. As expected, the Phos spectrum of $5 \mathrm{Cz}-\mathrm{TRZ}$ is well resolved and shows a characteristic vibrational structure, indicating that the $\mathrm{T}_{1}$ state is not a pure $\mathrm{CT}$ state but has LE character as well. In addition, the transient electron spin resonance (TrESR) measurements for 5Cz-TRZ showed relatively broad, polarized spin resonance signals that are indicative of $\mathrm{T}_{1}$ states formed following spin-orbit-coupling- 
mediated intersystem crossing ${ }^{47}$ (see Fig. 2c, and Supplementary Table S2 for more detailed discussion of the data). The $\mathrm{S}_{1}$ and $\mathrm{T}_{1}$ state energies of $5 \mathrm{Cz}-\mathrm{TRZ}$ are estimated to be 2.85 and $2.79 \mathrm{eV}$ from the onset of low-temperature PL and the first peak of Phos spectra, respectively, resulting in a $0.06 \mathrm{eV} \Delta E_{S_{1}-T_{1}}$ value. These experimental data thus point both to a small $\Delta E_{S_{1}-T_{1}}$ and to $S_{1}$ and $T_{1}$ states with different excitation characters, which is consistent with our theoretical results.

In order to evaluate the delayed fluorescence behaviour, the transient PL characteristics were analysed in degassed solutions at room temperature (Fig. 2d). 5Cz-TRZ exhibits clear prompt and delayed fluorescence components. As an example, the delayed exciton lifetime of $5 \mathrm{Cz}-\mathrm{TRZ}$ is as short as $0.8 \mu \mathrm{s}$ in dimethyl formamide. The $k_{\mathrm{RISC}}$ value was estimated from the PL quantum efficiencies (PLQE) and the exciton lifetime of the prompt and delayed components (a detailed description of the calculations is provided in the Supplementary Information). $5 \mathrm{Cz}$-TRZ shows an extremely fast $k_{\mathrm{RISC}}$ of $\sim 1.5 \times 10^{7} \mathrm{~s}^{-1}$ in toluene, which is some two orders of magnitude higher than those of conventional TADF molecules such as TmCz-TRZ and DACT-II (Supplementary Fig. S6 and Table S3). We also investigated the excited-state dynamics of 5Cz-TRZ using pump-probe transient absorption (TA) spectroscopy (Fig. 2e). The picosecond TA measurement spectra exhibit broad photoinduced absorption (PIA) signals over the entire spectral region $(500-1025 \mathrm{~nm})$; the signals undergo rapid spectral shifts within the first $10 \mathrm{ps}$, which can be assigned to structural and solvent reorganization processes $^{48}$ (more detailed discussions of the dynamical processes can be found in the Supplementary Information). Nanosecond TA was also performed to investigate the timescales of ISC/RISC and to characterize triplet excited-state features. As illustrated by Figs. 2f and $\mathrm{g}, 5 \mathrm{Cz}$-TRZ undergoes ISC from the ${ }^{1} \mathrm{CT}$ state to the triplet manifold with a time constant of $4.5 \mathrm{~ns}$, which is shorter than those of TmCz-TRZ and DACT-II (Supplementary Figs. S7 and 8). The near degenerate singlet and triplet states, together with the large spin- 
orbit couplings, are responsible for the rapid ISC times observed in 5Cz-TRZ. Time-resolved temperature-dependent PL decay data for $15 \mathrm{wt} \%$-doped films of $5 \mathrm{Cz}$-TRZ in $m \mathrm{CBP}$ were also collected to further investigate their excited-state decay kinetics (Fig. 2h). The RISC rates are observed to increase with increasing temperature from 200 to $300 \mathrm{~K}$ (Fig. $2 \mathbf{i})$ and reach $1.37 \times$ $10^{7} \mathrm{~s}^{-1}$ at $300 \mathrm{~K}$. The $\Delta E_{S_{1}-T_{1}}$ value estimated is $0.03 \mathrm{eV}$ from the Arrhenius plots of the $k_{\mathrm{RISC}}$ values in 5Cz-TRZ (Supplementary Fig. S5).

\section{Device characterization}

Given the promising photophysical properties of 5Cz-TRZ, which include fast $k_{\text {RISC }}$ and high PLQE, its EL performance was evaluated. The optimized device configuration shown in Fig. 3 has the following architecture: ITO/HAT-CN $(10 \mathrm{~nm}) / \alpha-\mathrm{NPD} \quad(30 \mathrm{~nm}) / \mathrm{Tris}-\mathrm{PCz}$ $(10 \mathrm{~nm}) / m \mathrm{CBP}(6 \mathrm{~nm}) / 15 \mathrm{wt} \%$ TADF emitter: $m$ CBP $(20 \mathrm{~nm}) / \mathrm{CF} 3-\mathrm{TRZ}(10 \mathrm{~nm}) / 30 \mathrm{wt} \% \mathrm{Liq}:$ BPPB (45 nm)/Liq (2 nm)/Al (120 nm), where HAT-CN, $\alpha$-NPD, Tris-PCz, $m$ CBP, CF3-TRZ and BPPB are 1,4,5,8,9,11-hexaazatriphenylene hexacarbonitrile, N,N'-diphenyl-N,N'-bis(1naphthyl)-1,10-biphenyl-4,4'-diamine, 9,9',9"-triphenyl-9H,9'H,9"'H-3,3':6'3"'tercarbazole,

3,3-di(9H-carbazol-9-yl)biphenyl,

phenylene))bis(2,4-diphenyl-1,3,5-triazine) and 1,3-bis(9-phenyl-1,10-phenanthrolin-2yl)benzene, respectively; $15 \mathrm{wt} \%$ of $5 \mathrm{Cz}$-TRZ doped in $m \mathrm{CBP}$ (device A) served as the emitting layer (EML). For comparison, the control devices (devices B and C) were fabricated with the TmCz-TRZ and DACT-II emitters. The materials used and the device structures are given in Supplementary Fig. S10.

Devices A and B show sky-blue emission with EL peaks at 486 and $496 \mathrm{~nm}$, respectively, whereas device $C$ exhibits pure green emission with the emission peak at $530 \mathrm{~nm}$ (Fig. 4b). As illustrated in Fig. 4a and Supplementary Fig. S11, high and stable efficiencies are achieved with device A. The maximum EQE of device A is $29.3 \%$, a value much higher 
than those of device B (19.7\%) and device C (24.3\%). It is especially noteworthy that device A, which has 5Cz-TRZ as dopant, exhibits an extremely low efficiency roll-off. For example, the efficiency remains at $28.6 \%$ at $1000 \mathrm{~cd} \mathrm{~m}^{-2}$ and still as high as $27.0 \%$ at $5000 \mathrm{~cd} \mathrm{~m}^{-2}$, corresponding to a $2.3 \%$ and $7.8 \%$ decrease in $\mathrm{EQE}$ at these brightness points. In contrast, devices B and C display severe efficiency roll-off with much lower EQEs of only $8.6 \%$ and $16.2 \%$ at $5000 \mathrm{~cd} \mathrm{~m}^{-2}$, which corresponds to $56.3 \%$ and $33.3 \%$ decreases in EQE, respectively.

The transient EL decay characteristics at different luminance levels were used to further confirm the low efficiency roll-off behaviour of the 5Cz-TRZ-based device (see Fig. 4c), with very little reduction in the excition lifetime observed at high luminance levels. With this impressive behaviour confirmed, we then analysed the operational stability of the devices (Fig. 4d), showing that the $T_{90}$ (time to reach $90 \%$ of initial luminance) values of devices A, B, and $\mathrm{C}$ are $35.4,3.2$, and $5.1 \mathrm{~h}$ for an initial brightness $\left(L_{0}\right)$ of $5000 \mathrm{~cd} \mathrm{~m}^{-2}$. Device A thus exhibited by far the longest operational stability, with device lifetime over ten times longer than that of device B. The $T_{90}$ of device A is predicted to be $592.2 \mathrm{~h}$ at $1000 \mathrm{~cd} \mathrm{~m}^{-2}$ according to the formula $\mathrm{LT}\left(L_{1}\right)=\mathrm{LT}\left(L_{0}\right) \times\left(L_{0} / L_{1}\right)^{n}$, where $L_{1}$ is the initial luminance of $1000 \mathrm{~cd} \mathrm{~m}^{-2}(n=1.75$, which is obtained by fitting $T_{90}$ versus $L_{0}$ in Supplementary Fig. S14) ${ }^{49}$ To the best of our knowledge, this result represents the longest device lifetime reported for TADF-based OLEDs (Supplementary Table S5).

Hole-only and electron-only devices based on the structures of devices A, B, and C were also fabricated to characterize the carrier-transport properties. All devices exhibited similar properties (Supplementary Fig. S15), which implies that charge-carrier transport is not the major factor determining the differences in efficiency roll-off and operational stability. Thus, the reduced efficiency roll-off and improved operational stability of device A is primarily determined by the fast $k_{\mathrm{RISC}}$ that limits the amount of bimolecular exciton annihilation processes taking place in the devices. 
To better understand the factors behind the high EQE of device A, we investigated the emitting-dipole orientations in these devices using variable-angle PL measurements. As shown in Supplementary Fig. S16, the orientation factor $(\theta)$ of $5 \mathrm{Cz}-\mathrm{TRZ}$ is estimated to be 0.17 (we recall that $\theta=0$ for completely horizontal alignment and 0.33 for isotropic distribution). ${ }^{50}$ Using this value, the optical simulation results indicate that device A can achieve a maximum EQE of $30.9 \%$, which is consistent with our experimental data.

The fast $k_{\mathrm{RISC}}$ of $5 \mathrm{Cz}-\mathrm{TRZ}$ inspired us to further investigate the performance of hyperfluorescent OLEDs exploiting 5Cz-TRZ as sensitizer (Supplementary Fig. S17). 2,5,8,11-tetra-tert-butylperylene (TBPe), 5,12-Dibutyl-1,3,8,10-tetramethylquinacridone (TMDQA), 2,8-di-tert-butyl-5,11-bis(4-tert-butylphenyl)-6,12-diphenyltetracene (TBRb) and 4-(Dicyanomethylene)-2-tert-butyl-6-(1,1,7,7-tetramethyljulolidin-4-yl-vinyl)-4H-pyran (DCJTB) were selected as sky-blue, green, yellow, and red fluorescent emitter dopants, respectively. Taking the same device structure as for the TADF-emitter-based OLEDs, the hyperfluorescent OLEDs based on 5Cz-TRZ as sensitizer show EQEs as high as $24.0 \%$ (device D; sky bule), 22.3\% (device E; green), 24.9\% (device F; yellow) and 23.5\% (device G; red). The slightly lower EQEs and larger efficiency roll-offs vs. the 5Cz-TRZ-emitter-based device are likely due to hole trapping by the fluorescent molecules, as the HOMO levels of the fluorescent materials are shallower compared to that of 5Cz-TRZ. However, the operational lifetimes of the OLEDs based on 5Cz-TRZ as the sensitizer were longer than that of the OLED with 5Cz-TRZ as the emitter dopant (see Fig. 4d). We do note that the EL spectrum of device D is somewhat different form its PL spectrum in the co-deposited film (Supplementary Fig. S18), which is most likely induced by the microcavity effect of the device rather than an incomplete energy transfer. ${ }^{21}$

Finally, we constructed a white OLED (device $\mathrm{H}$ ) by employing $5 \mathrm{Cz}-\mathrm{TRZ}$ as the skyblue emitter, complemented by TBRb and DCJTB as yellow and red emitters, respectively. A 
high EQE of $21.8 \%$ was achieved with Commission Internationale de I'Eclairage (CIE) coordinates of $(0.43,0.45)$ and colour rendering index (CRI) of 81 (Fig. 4b).

\section{Conclusion}

In summary, we have designed a sky-blue metal-free organic emitter (5Cz-TRZ) with a RISC rate as fast as $1.5 \times 10^{7} \mathrm{~s}^{-1}$, some two orders of magnitude higher than the typical values for TADF emitters. The introduction of multiple carbazole donors in $5 \mathrm{Cz}-\mathrm{TRZ}$ that form chargeresonance-type hybrid triplet states leads simultaneously to a small single-triplet energy splitting, large spin-orbit coupling, and dense triplet-state manifold, which all contribute to the fast RISC. In combination with a near 100\% PLQE and a strong horizontal dipole orientation in the doped film, state-of-the-art OLED devices exploiting 5Cz-TRZ as emitter show a maximum EQE of $29.3 \%$, a low efficiency roll-off at high luminance (e.g. a mere $2.3 \%$ rolloff of the EQE at $1,000 \mathrm{~cd} \mathrm{~m}^{-2}$ ), and an estimated $T_{90}$ value of $\sim 600 \mathrm{~h}$ for an initial brightness of $1000 \mathrm{~cd} \mathrm{~m}^{-2}$. Importantly, we note that this lifetime is tenfold longer than that of the reference device based on the TmCz-TRZ emitter, which has a slower $k_{\mathrm{RISC}}$. Additionally, fullcolour hyperfluorescent OLEDs exploiting $5 \mathrm{Cz}-\mathrm{TRZ}$ as a sensitizer reach high EQE values of over $22.0 \%$. Thus, we consider that our novel design strategy represents an important step towards achieving a fast $k_{\mathrm{RISC}}$ in TADF molecules, paving the way to efficient and stable TADF and hyperfluorescent OLEDs.

\section{Methods}

\section{Synthesis and characterization}

The general procedure for the synthesis of the TADF molecules and the characterization of their chemical properties are reported in the Supplementary Information.

\section{Computational details}


The initial ground-state geometries of the molecules were optimized with the long-range corrected functional $\omega$ B97XD (with the default range-separation parameter $\omega$ of $0.2 \mathrm{bohr}^{-1}$ ) and the $6-31 \mathrm{G}(\mathrm{d}, \mathrm{p})$ basis set. ${ }^{51}$ Then, following our earlier works, ${ }^{44,52}$ an iterative "gap-tuning" procedure was applied to obtain the optimal $\omega$ values for these geometries. The Tamm-Dancoff approximation (TDA) was employed in the framework of time-dependent density functional theory (TD-DFT) to study the excited-state properties; all the excited-state properties were examined at the TDA tuned- $\omega \mathrm{B} 97 \mathrm{XD} / 6-31 \mathrm{G}(\mathrm{d}, \mathrm{p})$ level combined with the polarizable continuum model (PCM; implicit solvent: toluene). Natural transition orbital analyses were also performed to examine the nature of the excited states; the spatial overlaps between the density distributions of the hole and electron wavefunctions in the excited states were estimated with the Multiwfn code. ${ }^{53}$ All quantum-chemical calculations were performed with the Gaussian 16 program. ${ }^{54}$ In addition, the spin-orbit couplings were estimated by employing the Breit-Pauli spin-orbit Hamiltonian with an effective charge approximation, as implemented in the PySOC code. ${ }^{55}$

\section{Absorption, steady-state PL spectra and PLQE measurements}

UV-vis and PL spectra were recorded on a Perkin-Elmer Lambda 950 KPA spectrophotometer and JobinYvon FluoroMax-3 fluorospectrophotometer, respectively. Phos spectra were recorded on a JASCO FP-6500 fluorescence spectrophotometer at 77 K. Absolute PL quantum yields were measured on a Quantaurus-QY measurement system (C11347-11, Hamamatsu Photonics) under nitrogen flow and all samples were excited at $360 \mathrm{~nm}$.

\section{Eectron paramagnetic resonance (EPR) measurements}

Transient CW EPR measurements (trEPR) were performed in the Centre for Advanced ESR (CAESR) in the Department of Chemistry of the University of Oxford, using both Bruker BioSpin EleXSys I E680 and EleXSys II E580 spectrometers at X-band, ca. 9.75 GHz. Details of the optical simulations are given in the Supplementary Information. 


\section{Time-resolved spectroscopic measurements}

All the solutions were deoxygenated with dry nitrogen gas to eliminate the deactivation of triplets by oxygen. The transient PL decay characteristics of solution samples were recorded using a Quantaurus-Tau fluorescence lifetime measurement system (C11367-03, Hamamatsu Photonics). Time-resolved PL of the film samples were measured using an Andor electrically gated intensified charge-coupled device and laser excitation at $350 \mathrm{~nm}$. For low-temperature measurements, an Oxford Instruments continuous flow cryostat was used with liquid helium as the coolant. Experimental details for the transient PL and absorption measurements are given in Supplementary Information.

\section{Transient absorption (TA) measurements}

Sample photoexcitation in the nanosecond transient absorption experiments was achieved by the third harmonic $(355 \mathrm{~nm})$ of an electronically triggered Q-switched $\mathrm{Nd}: \mathrm{YVO}_{4}$ laser $(\sim 1 \mathrm{~ns}$ pulse length, Advanced Optical Technologies Ltd AOT-YVO-25QSPX). For the picosecond transient absorption, $\sim 100 \mathrm{fs}$ excitation pulses at $400 \mathrm{~nm}$, generated from the second harmonic of the $800 \mathrm{~nm}$ fundamental of the Ti:sapphire laser (Spectra Physics Solstice Ace), was used. For both temporal regions, the probe was generated by home-built broadband visible (500 $770 \mathrm{~nm})$ and NIR (830 - $1025 \mathrm{~nm})$ non-collinear optical parametric amplifiers (NOPAs), pumped using the frequency-doubled output $(400 \mathrm{~nm})$ of the Ti:sapphire laser. The delay between the pump and probe pulses was varied using a Stanford DG645 delay generator for the nanosecond measurements, while a mechanical delay stage (Thorlabs DDS300-E/M) was used to delay the probe with respect to the pump for the picosecond measurements. The transmitted probe pulses were collected with a silicon dual-line array detector (Hamamatsu S8381-1024Q) which was driven and read out by a custom-built board from Stresing Entwicklungsbüro.

\section{Angle-dependent PL measurements}


The angle-dependent PL measurement was measured by a spectrometer (Ocean Optics USB 4000) together with a $405 \mathrm{~nm}$ continuous wave laser as the excitation source, a half cylindrical lens, a motorized rotation stage, and a polarizer to select $p$-polarized light. Details of the optical simulations are given in the Supplementary Information.

\section{Device fabrication and measurements}

OLEDs were fabricated through vacuum deposition of the materials at $c a .6 .0 \times 10^{-8}$ Torr onto ITO-coated glass substrates with a sheet resistance of ca. $15 \Omega / \square$. The ITO surface was sequentially cleaned ultrasonically with acetone, ethanol, and deionized water, dried in an oven, and then exposed to UV/ozone for about $30 \mathrm{~min}$. Organic layers were deposited at a rate of $2-$ $3 \AA$ As/ Liq was subsequently deposited at $0.2 \AA /$ s and then capped with $\mathrm{Al}$ (ca. $3 \AA$ $/ \mathrm{s}$ ). The devices were exposed once to nitrogen gas after the formation of the organic layers because a metal mask was included to define the cathode area. For all the OLEDs, the emitting area determined by the overlap of the two electrodes was $4.5 \mathrm{~mm}^{2}$. Current density-voltageluminescence $(J-V-L)$ characteristics were measured using a Keithley 2400 sourcemeter, Keithley 2000 multimeter and a calibrated silicon photodiode. Time-resolved EL of the samples was recorded by the same ICCD spectrometer used in the time-resolved PL measurements.

\section{Acknowledgements}

L.S.C., A.J.G., E.W.E. and R.H.F. acknowledge the Engineering and Physical Sciences Research Council (EPSRC) for funding (EP/M01083X/1, EP/M005143/1). The Centre for Advanced ESR (CAESR) is supported by UK EPSRC (EP/L011972/1). We thank Diamond Light Source (UK) for synchrotron beamtime on I19 (CY21497). X.-K.C. and J.-L.B. acknowledge support from the Georgia Institute of Technology, Georgia Research Alliance, Vasser-Woolley Foundation, and Kyulux. H.Y., Z.S.L., N.H. and C.A acknowledge the Japan Science and Technology Agency (JST), ERATO, Adachi Molecular Exciton Engineering 
Project for funding (JPMJER1305, Japan). S.-F.Z. acknowledges financial support by Guangxi Department of Science and Technology (NO.AD19110030), Department of Education (NO.2019KY0394) and the start-up funds provided by Guangxi University of Science and Technology. E.W.E also thanks the Leverhulme Trust for funding (ECF-2019-054). We also thank Prof. Jun Yeob Lee for providing the control TADF materials.

\section{Author Contributions}

L.S.C. designed the molecules and carried out device fabrication and measurements. A.J.G. conducted the transient absorption experiments. S.-F.Z. performed the theoretical calculations under the supervision of X.-K.C.. Y.L. performed the molecular orientation and optical simulations under the supervision of S. R.. H.Y. and Z.S.L carried out steady-state and timeresolved photophysical properties under the supervision of C.A.. E.W.E. and W.K.M conducted the transient electron spin resonance measurements and analysed the results. T.K.R. performed single crystal X-ray diffraction and analysed the results. H.N. participated in the discussion of the photophysical mechanism. L.S.C., A.J.G., X.-K.C., J.-L.B. and R.H.F. analysed the data and wrote the manuscript. All authors discussed the progress of research and reviewed the manuscript.

\section{Data availability}

The data that support the plots within this paper and other findings of this study are available from the corresponding author upon reasonable request.

\section{Competing interests}

The authors declare no competing interests. 


\section{References}

1. Baldo, M. A. et al. Highly efficient phosphorescent emission from organic electroluminescent devices. Nature 395, 151-154 (1998).

2. Einzinger, M. et al. Sensitization of silicon by singlet exciton fission in tetracene. Nature 571, 90-94 (2019).

3. Samuel, I. D. W. \& Turnbull, G. A. Organic Semiconductor Lasers. Chem. Rev. 107, 12721295 (2007).

4. Kohler, A. \& Bassler, H. Triplet states in organic semiconductors. Mater. Sci. Eng. R 66, 71-109 (2009).

5. Adachi, C., Baldo, M. A., Thompson, M. E. \& Forrest, S. R. Nearly $100 \%$ internal phosphorescence efficiency in an organic light emitting device. J. Appl. Phys. 90, 5048$5051(2001)$.

6. Ma, Y., Zhang, H., Shen, J. \& Che, C. Electroluminescence from triplet metal-ligand charge-transfer excited state of transition metal complexes. Synth. Met. 94, 245-248, (1998).

7. Köhler, A. \& Beljonne, D. The singlet-triplet exchange energy in conjugated polymers. Adv. Funct. Mater. 14, 11-18 (2004).

8. Kondakov, D. Y. Characterization of triplet-triplet annihilation in organic light-emitting diodes based on anthracene derivatives. J. Appl. Phys. 102, 114504 (2007).

9. Ly, K. T. et al. Near-infrared organic light-emitting diodes with very high external quantum efficiency and radiance. Nat. Photon. 11, 63-68 (2017).

10. Li, L. K. et al. Strategies towards rational design of gold(iii) complexes for highperformance organic light-emitting devices. Nat. Photon. 13, 185-191 (2019).

11. Hamze, R. et al. Eliminating nonradiative decay in $\mathrm{Cu}(\mathrm{I})$ emitters: $>99 \%$ quantum efficiency and microsecond lifetime. Science 363, 601-606 (2019). 
12. Ai, X. et al. Efficient radical-based light-emitting diodes with doublet emission. Nature 563, 536-540 (2018).

13. Uoyama, H., Goushi, K., Shizu, K., Nomura, H. \& Adachi, C. Highly efficient organic light-emitting diodes from delayed fluorescence. Nature 492, 234-238 (2012).

14. Zhang, Q. et al. Efficient blue organic light-emitting diodes employing thermally activated delayed fluorescence. Nat. Photon. 8, 326-332 (2014).

15. Liu, Y., Li, C., Ren, Z., Yan, S. \& Bryce, M. R. All-organic thermally activated delayed fluorescence materials for organic light-emitting diodes. Nat. Rev. Mater. 3, 18020 (2018).

16. Wong, M. Y. \& Zysman-Colman, E. Purely organic thermally activated delayed fluorescence materials for organic light-emitting diodes. Adv. Mater. 29, 1605444 (2017).

17. dos Santos, P. L. et al. Triazatruxene: A Rigid Central Donor Unit for a D-A 3 Thermally Activated Delayed Fluorescence Material Exhibiting Sub-Microsecond Reverse Intersystem Crossing and Unity Quantum Yield via Multiple Singlet-Triplet State Pairs. Adv. Sci. 5, 1700989 (2018).

18. Cui, L.-S. et al. Controlling synergistic oxidation processes for efficient and stable blue thermally activated delayed fluorescence devices. Adv. Mater. 28, 7620-7625 (2016).

19. Noda, H., Nakanotani, H. \& Adachi, C. Excited state engineering for efficient reverse intersystem crossing. Sci. Adv. 4, eaao6910 (2018).

20. Cui, L.-S. et al. Long-lived efficient delayed fluorescence organic light-emitting diodes using n-type hosts. Nat. Commun. 8, 2250 (2017).

21. Chan, C.-Y. et al. Efficient and stable sky-blue delayed fluorescence organic light-emitting diodes with CIEy below 0.4. Nat. Commun. 9, 5036 (2018).

22. Turro, N. J., Ramamurthy, V. \& Scaiano, J. C. in Principle of Molecular Photochemistry: An Introduction Ch. 3, 113-118 (University Science Books, 2009). 
23. Gómez-Bombarelli, R. et al. Design of efficient molecular organic light-emitting diodes by a high-throughput virtual screening and experimental approach. Nat. Mater. 15, 1120-1127 (2016).

24. Kondo1, Y. et al. Narrowband deep-blue organic light-emitting diode featuring an organoboron-based emitter. Nat. Photon. 13, 678-682 (2019).

25. Kotadiya, N., Blom, P. W. M. \& Wetzelaer, G.-J. A. H. Efficient and stable single-layer organic light-emitting diodes based on thermally activated delayed fluorescence. Nat. Photon. 13, 765-769 (2019).

26. Ahn, D. H. et al. Highly efficient blue thermally activated delayed fluorescence emitters based on symmetrical and rigid oxygen-bridged boron acceptors. Nat. Photon. 13, 540-546 (2019)

27. Wu, T.-L. et al. Diboron compound-based organic light-emitting diodes with high efficiency and reduced efficiency roll-off. Nat. Photon. 12, 235-240 (2018).

28. Lee, D. R. et al. Design strategy for $25 \%$ external quantum efficiency in green and blue thermally activated delayed fluorescent devices. Adv. Mater. 27, 5861-5867 (2015).

29. Wu, K. et al. De novo design of excited-state intramolecular proton transfer emitters via a thermally activated delayed fluorescence channel. J. Am. Chem. Soc. 140, 8877-8886, (2018).

30. Pershin, A. et al. Highly emissive excitons with reduced exchange energy in thermally activated delayed fluorescent molecules. Nat. Commun. 10, 597 (2019).

31. Sharma, N. et al. Turn on of sky-blue thermally activated delayed fluorescence and circularly polarized luminescence (CPL) via increased torsion by a bulky carbazolophane donor. Chem. Sci. 10, 6689-6696 (2019). 
32. Lin, T.-A. et al. Sky-blue organic light emitting diode with $37 \%$ external quantum efficiency using thermally activated delayed fluorescence from spiroacridine-triazine hybrid. Adv. Mater. 28, 6976-6983 (2016).

33. Zhang, Y. et al. Multi-resonance induced thermally activated delayed fluorophores for narrowband green OLEDs. Angew. Chem. Int. Ed. 131, 1-7 (2019).

34. El-Sayed, M. A. The radiationless processes involving change of multiplicity in the diazenes. J. Chem. Phys. 36, 573-574 (1962).

35. Olivier, Y. et al. Nature of the singlet and triplet excitations mediating thermally activated delayed fluorescence. Phys. Rev. Mater. 1, 75602 (2017).

36. Marian, C. M. Spin-orbit coupling and intersystem crossing in molecules. WIREs Comput. Mol. Sci. 2, 187-203, (2012).

37. Etherington, M. K., Gibson, J., Higginbotham, H. F., Penfold, T. J. \& Monkman, A. P. Revealing the spin-vibronic coupling mechanism of thermally activated delayed fluorescence. Nat. Commun. 7, 13680 (2016).

38. Penfold, T. J., Gindensperger, E., Daniel, C. \& Marian, C. M. Spin-vibronic mechanism for intersystem crossing. Chem. Rev. 118, 6975-7025 (2018).

39. Murawski, C., Leo, K. \& Gather, M. C. Efficiency roll-off in organic light-emitting diodes. Adv. Mater. 25, 6801-6827 (2013).

40. Einzinger, M. et al. Shorter exciton lifetimes via an external heavy-atom effect: alleviating the effects of bimolecular processes in organic light-emitting diodes. Adv. Mater. 29, 1701987 (2017).

41. Reineke, S., Schwartz, G., Walzer, K. \& Leo, K. Reduced efficiency roll-off in phosphorescent organic light emitting diodes by suppression of triplet-triplet annihilation. Appl. Phys. Lett. 91, 123508 (2007). 
42. Lee, J. et al. Hot excited state management for long-lived blue phosphorescent organic light-emitting diodes. Nat. Commun. 8, 1-9 (2017).

43. Munkhbat, B., Wersäll, M., Baranov, D. G., Antosiewicz, T. J. \& Shegai, T. Suppression of photo-oxidation of organic chromophores by strong coupling to plasmonic nanoantennas. Sci. Adv. 4, eaas9552 (2018).

44. Chen, X.-K. et al. A new design strategy for efficient thermally activated delayed fluorescence organic emitters: from twisted to planar structures. Adv. Mater. 29, 1702767 (2017).

45. Chen, X. K., Zhang, S. F., Fan, J. X. \& Ren, A. M. Nature of highly efficient thermally activated delayed fluorescence in organic light-emitting diode emitters: nonadiabatic effect between excited states. J. Phys. Chem. C 119, 9728-9733 (2015).

46. Noda, H., Chen, X. K. et al. Critical role of intermediate electronic states for spin-flip processes in charge-transfer-type organic molecules with multiple donors and acceptors. Nat. Mater. 18, 1084-1090 (2019).

47. Evans, E. W. et al. Vibrationally assisted intersystem crossing in benchmark thermally activated delayed fluorescence molecules. J. Phys. Chem. Lett. 9, 4053-4058 (2018).

48. Kuang, Z. R. et al. Conformational Relaxation and thermally activated delayed fluorescence in anthraquinone-based intramolecular charge-transfer compound. J. Phys. Chem. C 122, 3727-3737, (2018).

49. Zhu, Z.-Q., Klimes, K., Holloway, S. \& Li, J. Efficient cyclometalated platinum(II) complex with superior operational stability. Adv. Mater. 29, 1605002 (2017).

50. Yokoyama, D. Molecular orientation in small-molecule organic light-emitting diodes. $J$. Mater. Chem. 21, 19187-19202 (2011). 
51. Chen, X.-K., Kim, D. \& Bredas, J.-L. Thermally activated delayed fluorescence (TADF) path toward efficient electroluminescence in purely organic materials: molecular level insight. Acc. Chem. Res. 51, 2215-2224 (2018).

52. Chen, X.-K. et al. Intramolecular Noncovalent Interactions Facilitate Thermally Activated Delayed Fluorescence (TADF). J. Phys. Chem. Lett. 10, 3260-3268 (2019).

53. Lu T. \& Chen F. Multiwfn: A multifunctional wavefunction analyser. J. Comput. Chem. 33, 580-592 (2012).

54. Frisch, M. J. et al. Gaussian, Inc., Wallingford CT, 2016.

55. Gao, X. et al. Evaluation of Spin-Orbit Couplings with Linear-Response Time-Dependent Density Functional Methods. J. Chem. Theory Comput. 13, 515-524 (2017). 
a
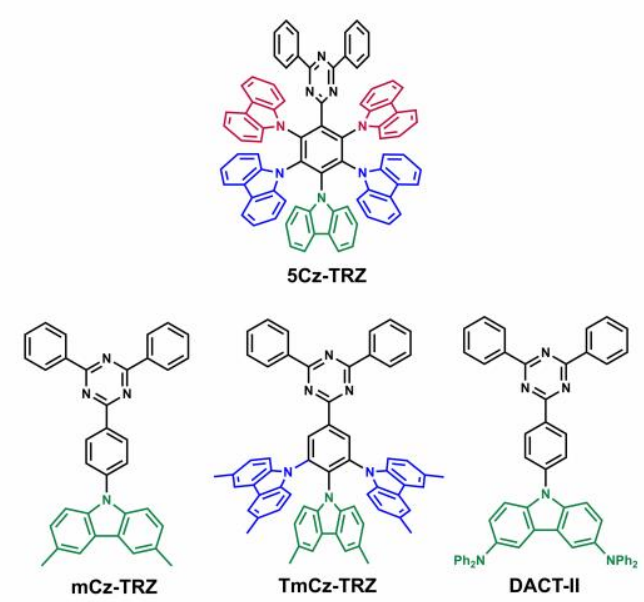

C

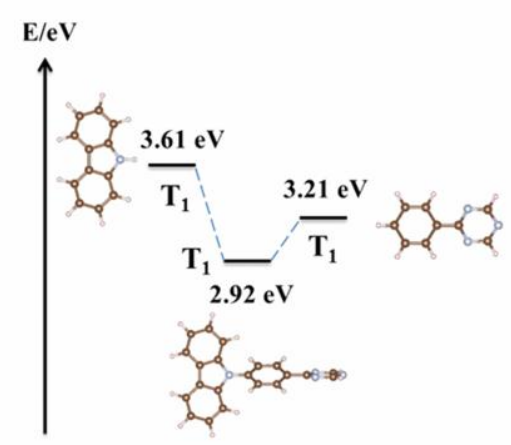

b
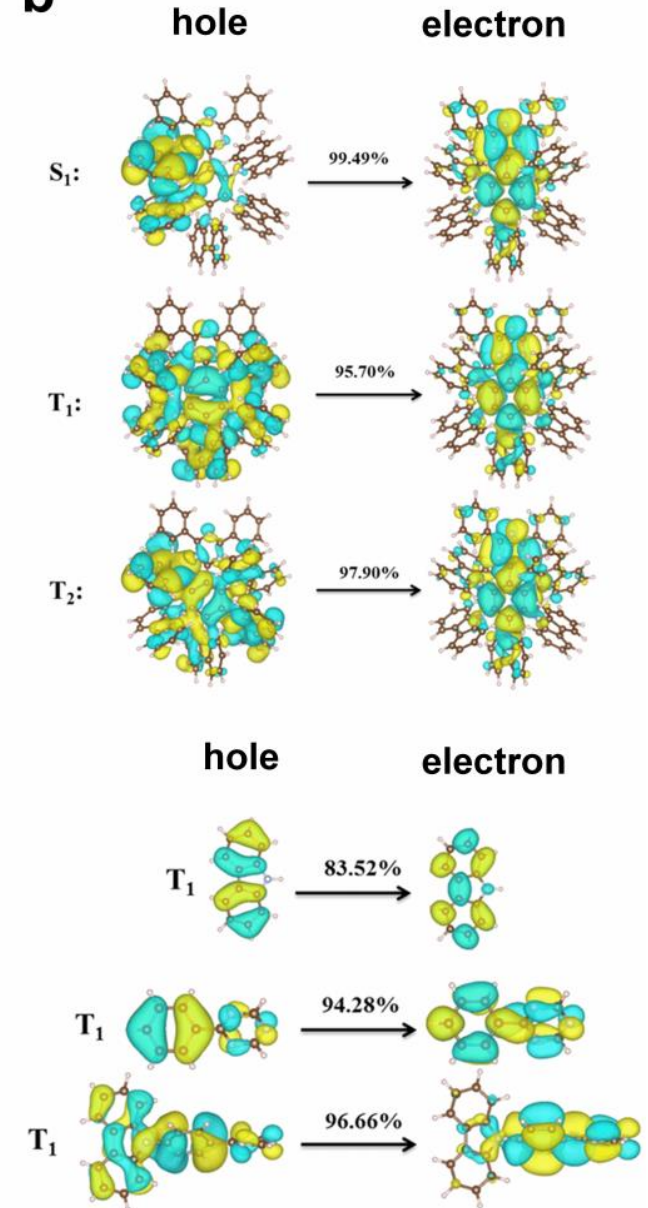

Fig. 1 | Chemical structures and results of quantum-chemical calculations for 5Cz-TRZ, mCz-TRZ, TmCz-TRZ, and DACT-II. (a) Chemical structures of 5CZ-TRZ, mCz-TRZ, TmCz-TRZ, and DACT-II. (b) Natural Transition Orbitals describing the excitation characters of the $S_{1}, T_{1}$, and $T_{2}$ states in 5Cz-TRZ; the weights of the hole-electron contributions to the excitations are included. (c) Left: Correlation diagram for the triplet states of the carbazole, phenylene-triazine, and carbazole-phenylene-triazine fragments, based on the $\mathrm{T}_{1}$ equilibrium geometry of $5 \mathrm{Cz}$-TRZ. Right: Natural Transition Orbitals for the $\mathrm{T}_{1}$ states in the three fragments. 
a

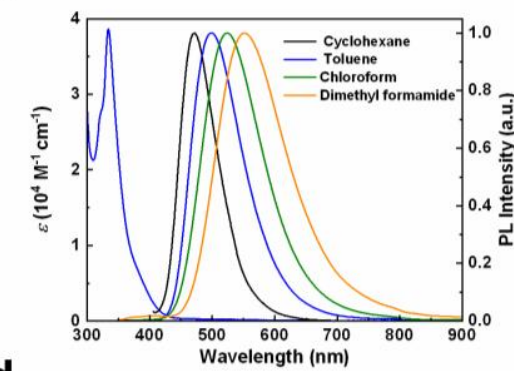

d

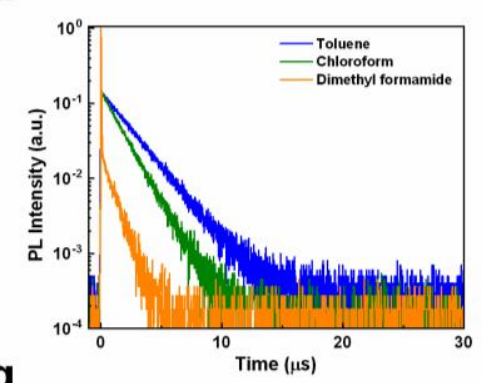

g

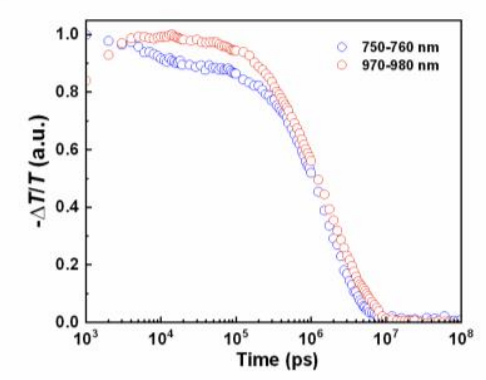

b

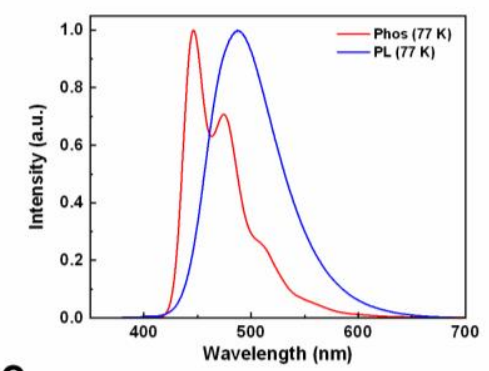

e
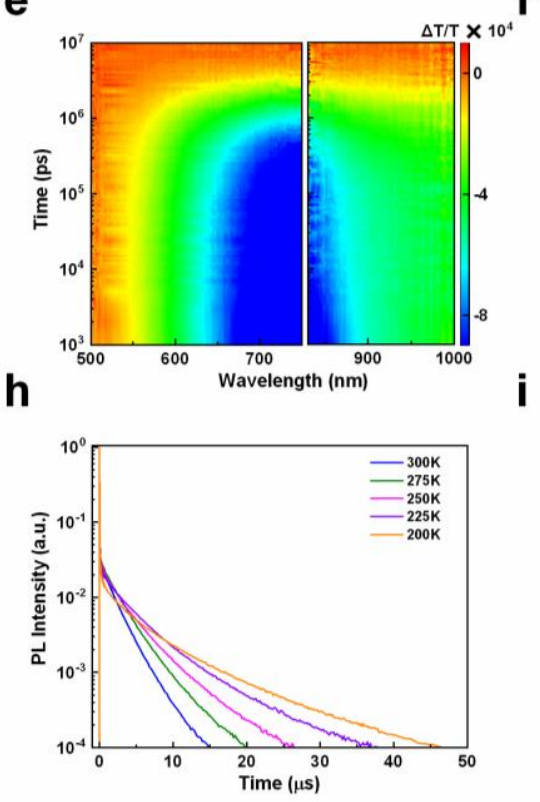

C
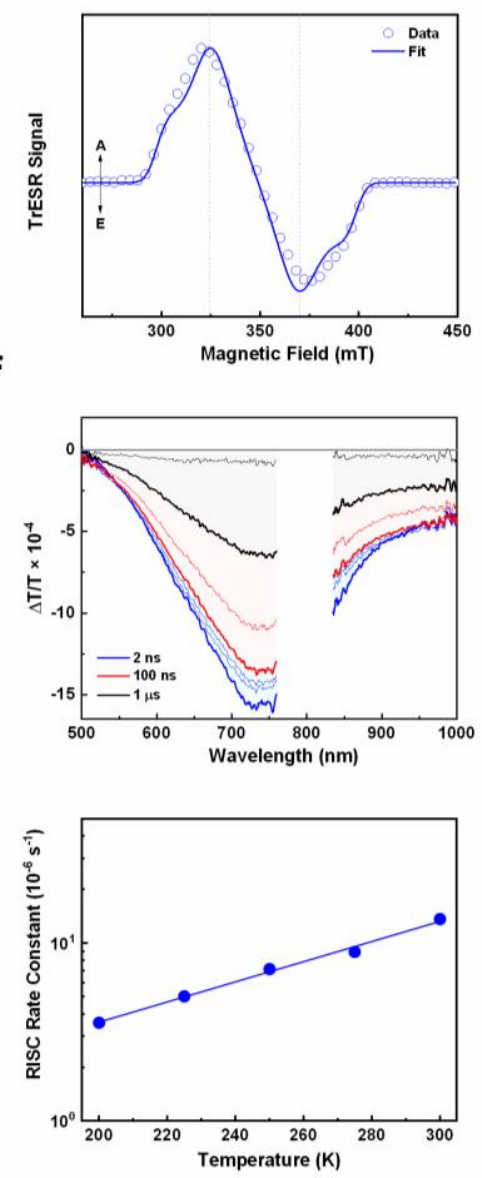

Fig. 2 | Photophysical characterization of 5Cz-TRZ. a, Absorption and PL spectra (300 K) of 5Cz-TRZ in dilute solution (toluene solvent). b, Phosphorescent (Phos) and PL spectra of 5Cz-TRZ in toluene glass at $77 \mathrm{~K}$. c, TrESR spectra $(80 \mathrm{~K})$ of $5 \mathrm{Cz}-\mathrm{TRZ}$ in dilute toluene solutions. d, Transient PL spectra $(300 \mathrm{~K})$ of 5Cz-TRZ in oxygen-free dilute solution with different polarity. e, Transient absorption contour maps $(300 \mathrm{~K})$ of $5 \mathrm{Cz}-\mathrm{TRZ}$ on nanosecond timescales in oxygen-free toluene. Excitation wavelength: $355 \mathrm{~nm}$, fluence: $5.49 \mu \mathrm{J} / \mathrm{cm}^{2}$. f, Transient absorption spectra $(300 \mathrm{~K})$ of $5 \mathrm{Cz}-\mathrm{TRZ}$ on nanosecond timescales in oxygen-free toluene. g, Decay kinetics of the transient absorption taken at 750-760 nm and 970-980 nm. h, Temperature-dependent transient PL (200-300 K) decay curves of the 5Cz-TRZ doped film. i, Temperature-dependent reverse intersystem crossing (RISC) rate constants of the 5Cz-TRZ doped film. 
a

\begin{tabular}{|c|}
\hline $\begin{array}{c}\text { Al } \\
\text { Liq } 2 \mathrm{~nm}\end{array}$ \\
\hline 30 wt\% Liq: BPPB $45 \mathrm{~nm}$ \\
\hline CF3-TRZ $10 \mathrm{~nm}$ \\
\hline EML $20 \mathrm{~nm}$ \\
\hline mCBP $6 \mathrm{~nm}$ \\
\hline Tris-PCz $10 \mathrm{~nm}$ \\
\hline a-NPD $30 \mathrm{~nm}$ \\
\hline HAT-CN $10 \mathrm{~nm}$ \\
\hline ITO \\
\hline
\end{tabular}

Device A: 15 wt\% 5Cz-TRZ: mCBP Device B: 15 wt\% TmCz-TRZ: mCBP Device C: 15 wt\% DACT-II: mCBP

Device D: 20 wt\% 5Cz-TRZ: 1 wt\% TBPe:mCBP

Device E: 20 wt\% 5Cz-TRZ: 1 wt\% TMDQA: mCBP

Device F: 20 wt\% 5Cz-TRZ: 1 wt $\%$ TBRb: $m$ CBP

Device G: 20 wt\% 5Cz-TRZ: 1 wt $\%$ TMDQA: mCBP

Device A-G

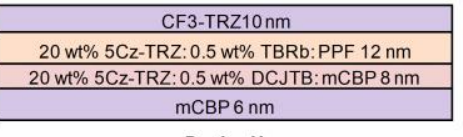

Device H b

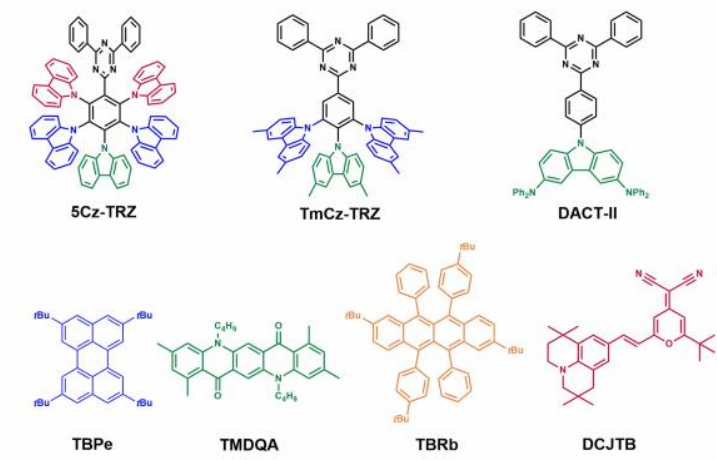

Fig. 3 Device structures and materials. a, Architectures of the TADF and hyperfluorescent

OLEDs. b, Chemical structures of the TADF and fluorescent emitters. 
a

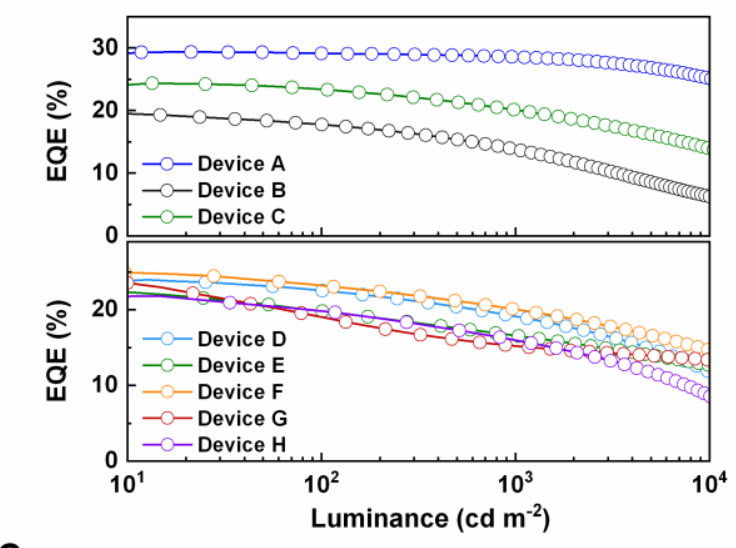

C

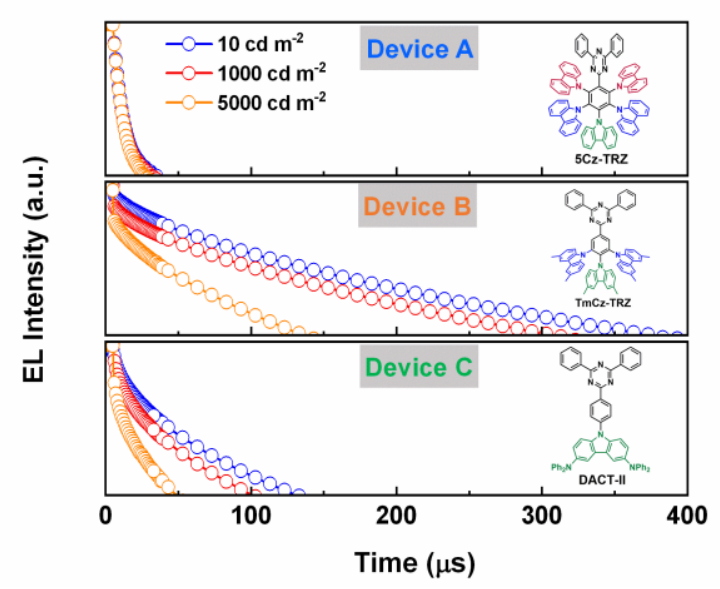

b

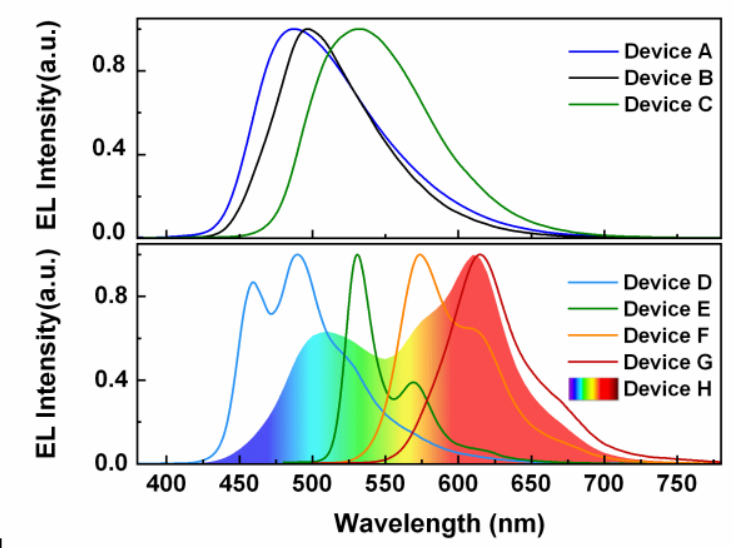

d

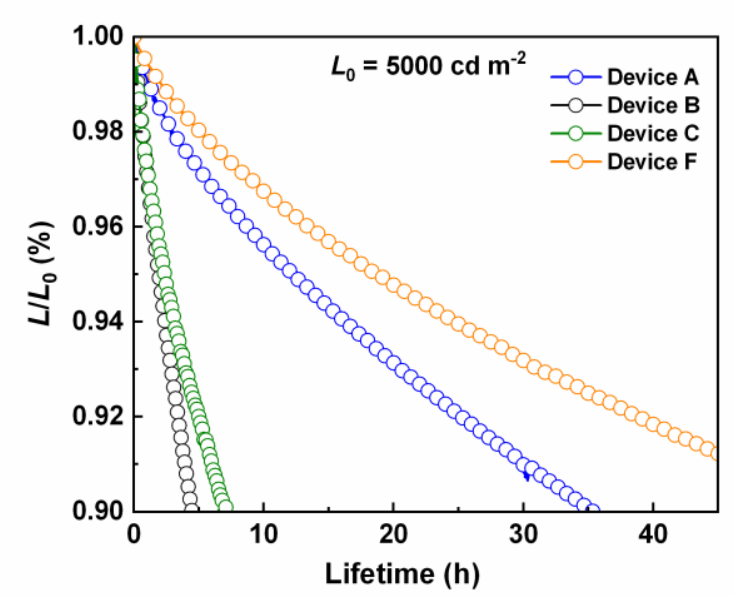

Fig. 4 | Device performance of OLEDs. a, EQE versus luminance characteristics. b, Normalized EL spectra. c, Transient EL decay characteristics of device A, B, C at 10, 1000, $5000 \mathrm{~cd} \mathrm{~m}^{-2}$. d, Operational lifetimes of the TADF and hyperfluorescent OLEDs. 